\title{
Laparoscopic total hysterectomy still not routinely chosen Operative description and available instruments
}

\author{
Alexandra Popa', Catalin Copaescu², Valerica Horhoianu ${ }^{1}$ \\ 1. Obstetrics \& Gynecology Department, Emergency University Hospital, Bucharest, Romania \\ 2. General Surgery Department, Ponderas Hospital, Bucharest, Romania \\ Corresponding Author: \\ Alexandra Popa \\ Emergency University Hospital \\ Splaiul Independentei Street, no. 169 \\ 050098 Bucharest, Romania \\ E-mail: md.alexandra.popa@gmail.com
}

Received: June 5th, 2019 - Accepted: July 24th, 2019

\begin{abstract}
Hysterectomy is the most common gynecological surgical intervention; therefore, there are many technical variations in different healthcare systems around the world. We aimed to review, step by step, the technique of laparoscopic hysterectomy as well as to present the available variety of surgical instruments impartially so that the operative team can decide in an informed manner the model and characteristics of the equipment used. The surgical technique is presented based on the experience of the authors, focusing mainly on intraoperative recommendation and suggestions. Advantages and disadvantages of the available instruments are also extensively detailed. Surgical positioning, as well as inserting the uterine manipulator are essential steps. The open technique is used to create pneumoperitoneum. The utero-ovarian ligament or the infundibulopelvic ligament is identified, coagulated and cut. The round ligament is incised, entering the space between the two layers of the broad ligament and advancing caudally in this space, which, if correctly identified, should be avascular. The uterine vessels located on the posterior sheet of the broad ligament are dissected and coagulated. The vaginal wall is sectioned with the help of the manipulator's cap, making it easier to expose the insertion line of the vagina on the cervix. The uterus is removed through the vagina or through a trans-parietal incision. Thereafter, the vagina is sutured using separate Vicryl sutures. Between 2011 and 2016, laparoscopic hysterectomy had an increasing trend all over Europe. With a reported percentage of $3 \%$, Romania ranks last in hysterectomies performed laparoscopically. The laparoscopic approach offers the advantages of minimal invasiveness: less pain, faster recovery and early social reintegration; therefore, this trend of improvement should become more accepted.
\end{abstract}

Keywords: total hysterectomy, laparoscopy, minimally invasive surgery

\section{Introduction}

Hysterectomy is the most common gynecological surgical intervention, and there are evident technical variations regarding the procedure in different healthcare systems around the world. Although there is a tendency to decrease the number of hysterectomies in western countries due to the extension of the conservative medical approach, it remains a procedure with a particularly important impact, both on the economy and quality of life of a large segment of the female population. Currently, hysterectomy is the eighth most frequent intervention performed in the healthcare systems in Europe. In terms of incidence, figures are reported in the literature varying from 5.4 hysterectomies/1000 women in the USA to $3.7 / 1000$ in Italy, 1.8/1000 in Denmark and 2.1/1000 in Sweden [1-4]. It is considered that the rate of hysterectomies relative to the number of females has decreased by about $1 \%$ per decade since the 1980s. Even considering these epidemiological aspects, it is estimated that approximately $20 \%$ of women will be hysterectomized before 70 years of age [5-8].

Regarding the surgical approach, the Statistical Office of The European Communities (Eurostat) reported in 2016 that more than $50 \%$ of all hysterectomies performed in Finland, Poland, the Czech Republic, Slovakia, Estonia and Belgium were performed laparoscopically, while lower rates of $33-40 \%$ for this approach were recorded in the Netherlands, Austria, France, Lithuania and Germany. In the rest of the European countries, even a lower number of laparoscopic hysterectomies were recorded, with the lowest figures in Croatia and Malta (9\%) and Romania (3\%) [9].

While analyzing these numbers, it is difficult to explain how laparoscopic cholecystectomy became a "gold standard" in benign gallbladder pathology in Romania, 
but the rest of the surgical procedures using minimally invasive techniques did not benefit from the same rate of development and implementation.

The lack of wide-spread techniques and familiarization with the laparoscopic instruments in benign hysterectomy can be easily noticed considering the presented numbers.

Literature studies have identified the following factors limiting the wide acceptance and implementation of laparoscopic hysterectomy: insufficient experience and training, lacking necessary equipment and support from colleagues [10].

The objective of this paper is to review the steps of the laparoscopic hysterectomy technique as well as to present the available variety of surgical instruments impartially so that the operative team can decide in an informed manner the model and characteristics of the equipment used.

\section{Surgical technique and laparoscopic instrumentation}

\section{Positioning the patient on the operating table}

The lithotomy position, with the patient on her back and legs abducted, similar to gynecologic examining position but keeping an open-angle about 150 degrees between the thighs and body, as well as between the calves and thighs. During the intervention, the patient is placed in the Trendelenburg position in order to mobilize the small bowel loops from the pelvis and visualize the structures of the reproductive apparatus.

The use of pneumatic or foam mattress for leg and shoulder support has been shown to be associated with a low risk of peripheral neurological injury and low postoperative pain at this level [11]. Also, the use of the pneumatic mattress prevents the patient from sliding off the surgical table.

The technique continues with the introduction of the uterine manipulator, without which the operation cannot be completed. There are many available options:

a) SecuFix produced by Richard Wolf (fig. 1) which has a universal tip (adjustable for several dimensions, depending on the size of the cervix) that is illuminated, facilitating the visualization portion where the vagina surrounds the cervix.

b) a classic reusable manipulator like that proposed by Karl Storz (fig. 2): ceramic caps of various sizes and a metal handle that are simple to use;

c) Rumi II - golden standard in the United States (fig.3), has caps of various sizes that adapt to the diameter of the cervix, an intrauterine balloon that prevents accidental extraction of the manipulator during surgery, the shaft allowing a full 140 degrees of articulation at the cervix, facilitating a faster dissection of the uterus.

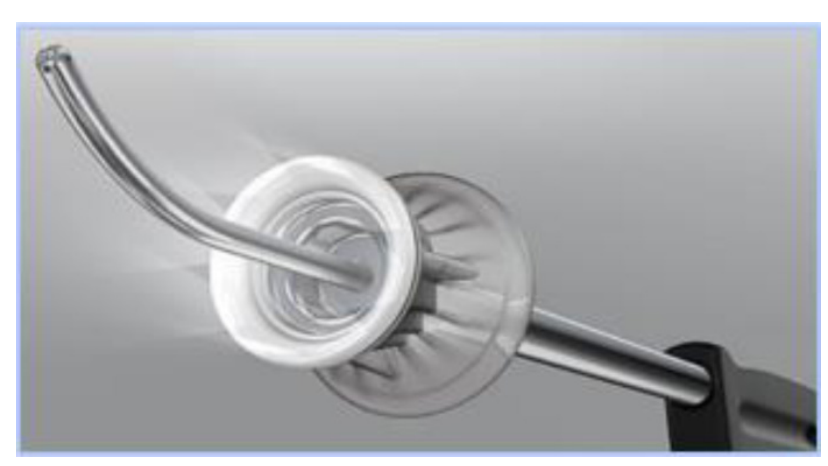

Figure 1

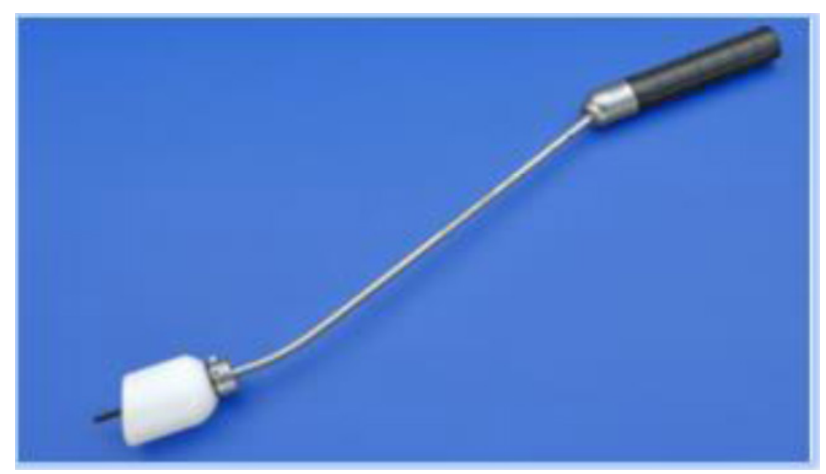

Figure 2

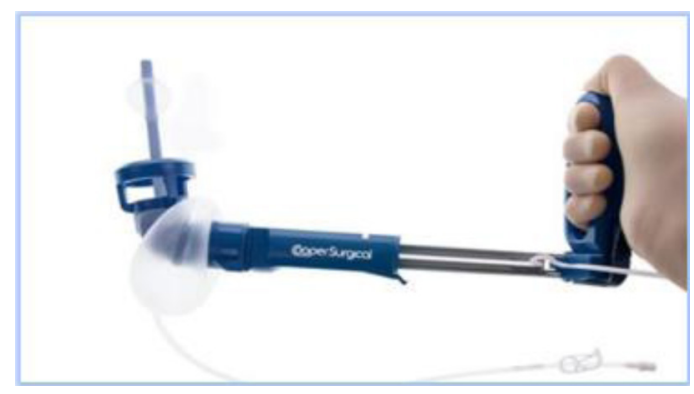

Figure 3

The use of any of the variants of uterine manipulators is the choice of the operating team, with the mention that the chosen one should have an adjustable cap that should be chosen according to the size of the cervix in order to avoid ureteral lesions (in the case of large caps) and difficult delimitation of the portion where the vagina surrounds the cervix (in the case of small caps).

When performing pneumoperitoneum, using an open supraumbilical technique is preferred to blind access (the use of a Veress needle). The $10 \mathrm{~mm}$ trocar is inserted, and the gas is used for the insufflation of the abdominal cavity, typically $12 \mathrm{~mm} \mathrm{Hg}$. The trocar can be metallic, reusable, or disposable. If disposable trocars are available, it is recommended to use an optical trocar that is equipped with an inflatable balloon (Applied Medical), which prevents the accidental extraction of the trocar when handling the optical camera (fig.4) 
Under visual control, the following trocars are introduced: two 5-mm trocars are placed bilaterally, laterally from the umbilicus, at the intersection between the spinoumbilical line with the midclavicular line, and another $5-\mathrm{mm}$ trocar is positioned in the midline, $3-4 \mathrm{~cm}$ above the pubic symphysis. It can also be positioned in the right iliac fossa, laterally to the rectus abdominis muscle (fig. 5). Choosing the position of the fourth trocar depends on the operator's will and experience; there are authors who believe that the latter variant is more ergonomic and more favorable when suturing the vagina [12].

If the uterus is very voluminous and cannot be extracted through the vagina, one of the $5-\mathrm{mm}$ trocars placed laterally to the umbilicus will be replaced by a 12-mm trocar to allow the use of a morcellator, usually on the left side. Metallic, reusable or plastic trocars can be used.

Ethicon trocars are provided with a sliding blade at the tip, which helps transfascial penetration. (fig. 5) Their insertion should be done with extreme caution in order to avoid iatrogenic vascular or intestinal lesions. Medtronic trocars are represented by a plastic, less sharp, bladeless tip, which reduces the risk of injury at the time of insertion (fig. 6). The third category is represented by plastic trocars with balloon tips, which also prevent their accidental extraction during the operation (Applied Medical, fig. 4).

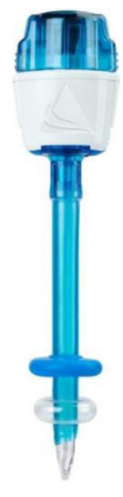

Figure 4: Applied Medical trocar

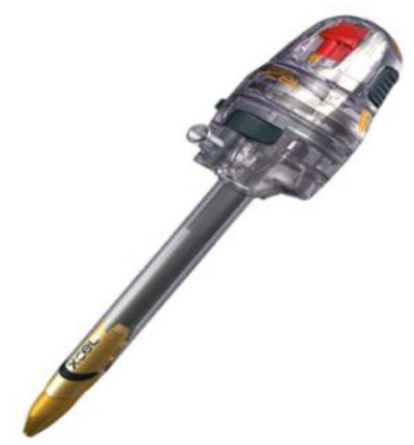

Figure 5: Ethicon trocar

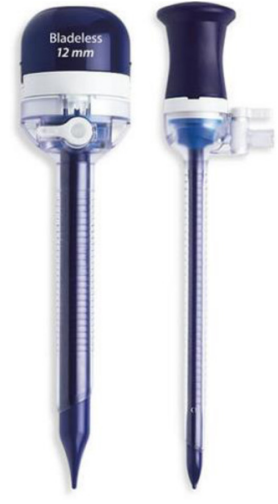

Figure 6: Medtronic trocars

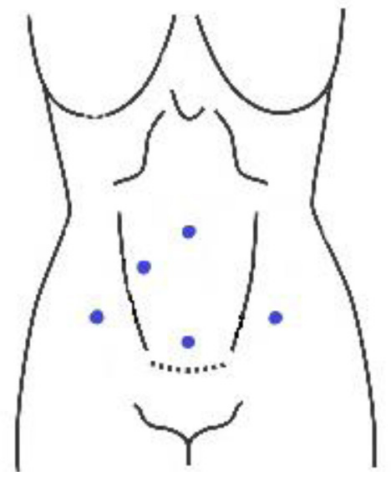

Figure 5: Anterior abdominal wall and trocar positioning in laparoscopic hysterectomy [13].

The surgical technique is largely standardized, being a mirror-like intervention: it can start on the right, and then the same steps are repeated on the left. The team member coordinating the uterine manipulator should push the uterus to the left of the patient so that the right flank is visible (Image 1), continuing in order to identify the following anatomical elements: the ovary and uterus, ureter, suspensory ligament of the ovary (infundibulopelvic ligament), round ligament and the vesico-uterine pouch.

It also should be decided which kind of high energy (ultrasonic coagulation and cutting) device will be used. Two broad categories are available:

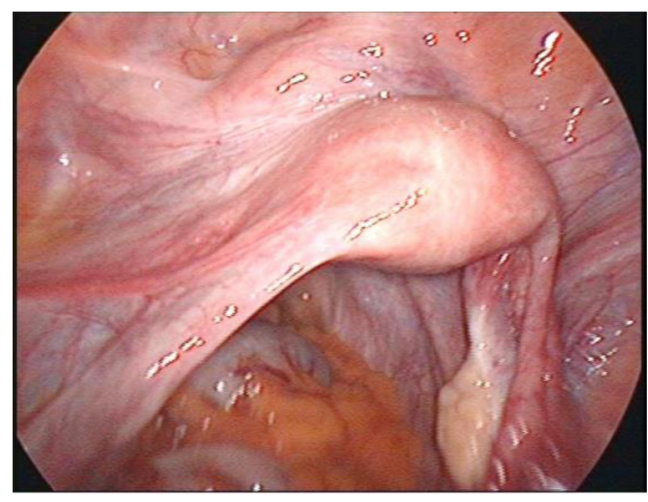

Image 1: Manipulation of the uterus while identifying the key anatomical structures. Personal collection. 
1. Bipolar current devices: LigaSure - Medtronic (fig. 7), Caiman - Aesculap (fig. 8), EnSeal -Ethicon (fig. 9) or BiCision - Erbe Elektromedizin (fig. 10).

2. Radiofrequency devices: Harmonic Ace 7 - Ethicon (Fig. 11), Sonicision Cordless Ultrasonic Dissection Device - Medtronic (Figure 12) or Thunderbeat - Olympus (Fig. 13)

Instruments in the first category can be used on vessels up to $7 \mathrm{~mm}$ in diameter with minimal Stergem postoperative bleeding risk. All these tools have similar features, and the operator's option for one of the variants is a matter of preference and availability. Two of them (Caiman and EnSeal) have a flexible tip that can be oriented very easily, which is a significant feature, of great technical help. In the case of a larger uterus with difficult access to the deep

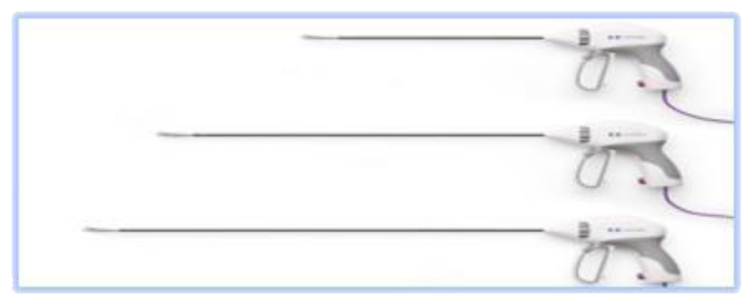

Figure 7

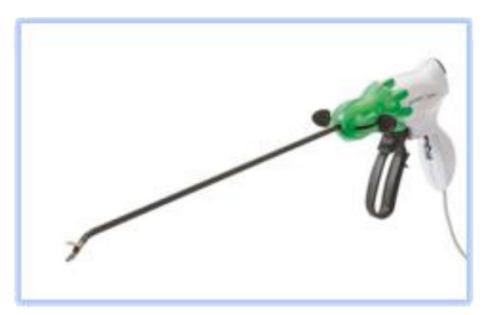

Figure 8

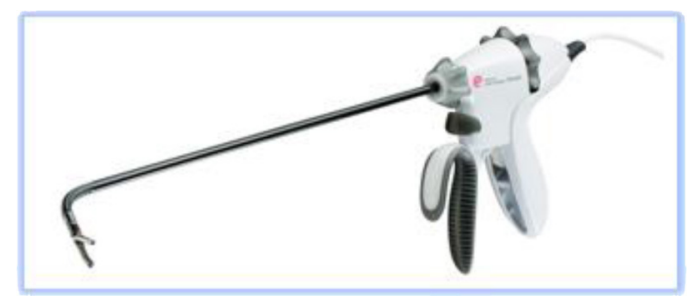

Figure 9

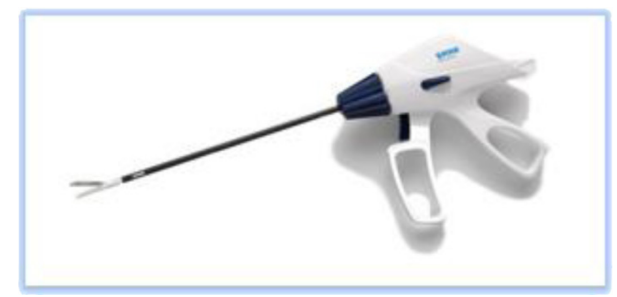

Figure 10

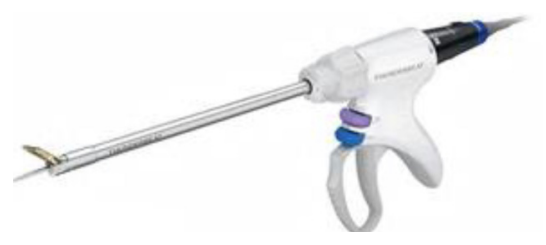

Figure 11

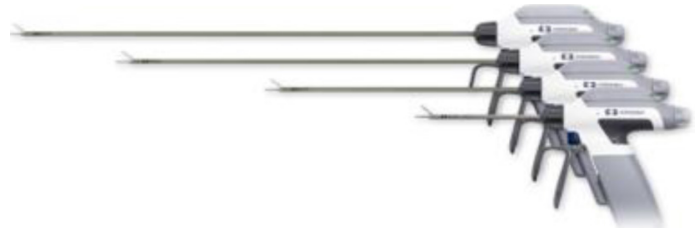

Figure 12

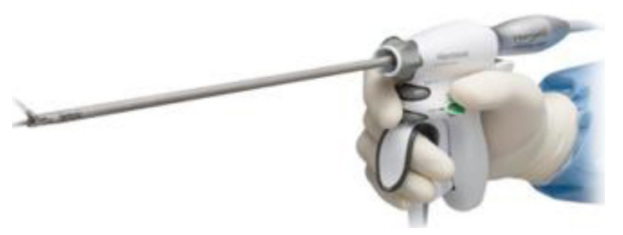

Figure 13

pelvis and cervix, it is preferable to choose such a flexible, high-energy device.

The instruments in the second category can mostly be used on vessels up to $5 \mathrm{~mm}$ in diameter (additional attention should be paid to the uterine artery) and produce a combination of vapor and smoke during use, which can be disturbing in some situations. Stergem complet propozitia aceasta. Also, it should be noted that Sonicision is batteryoperated and therefore portable and more ergonomic in use.

In the beginning, the utero-ovarian ligament (in case of simple hysterectomy) or the infundibulopelvic ligament (if salpingo-oophorectomy is associated) are identified, coagulated and cut (Image 2). For this step, as well as for the whole operation, either of the tools described in the two categories can be used. Once again, it is a matter of personal preference when choosing the devices used during the procedure, this paper aiming at an impartial presentation of the technological arsenal available at this time. The dissection advances with the coagulation and incision of the round ligament, the next step being to identify the space between the two layers of the broad ligament and advance caudally in this space, which, if correctly identified, should be avascular (Image 3).

The anterior layer should be coagulated and dissected step by step, progressing caudally towards the uterine vessels located on the posterior sheet of the broad ligament. Rigorous identification is recommended; only after that, dissection and coagulation should be performed. Coagulation of the vascular elements using the high 


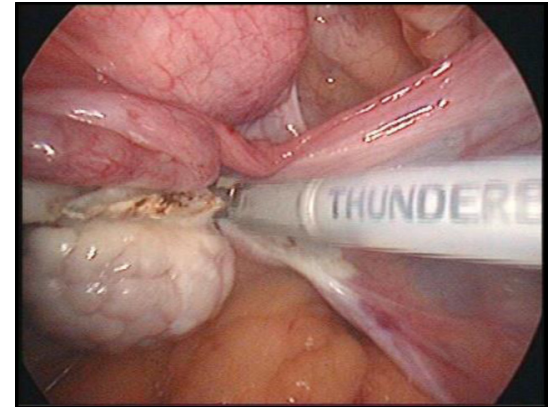

Image 2: Coagulation of the infundibulopelvic ligament. Personal collection

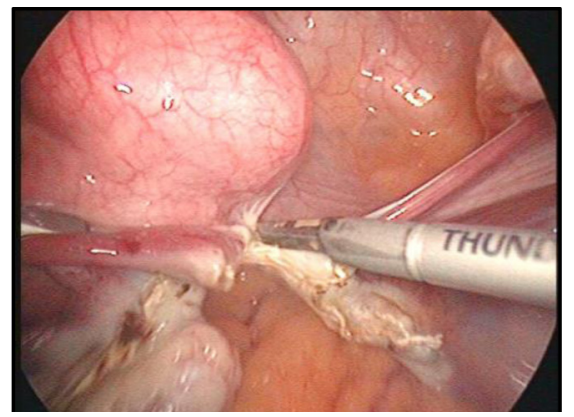

Image 3: Dissection of the broad ligament. Personal collection

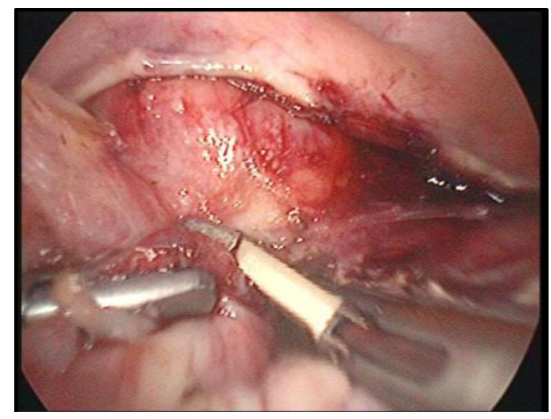

Image 4: Coagulation of vascular elements on the right side. Personal collection

energy device is done at least $1 \mathrm{~cm}$ above the incision point of the pubocervical fascia. During this procedure, the role of the uterine manipulator is essential; it should be oriented to the contralateral side towards the flank in which the operation is performed (Image 4). Everything should be done as close as possible to the uterus, minimizing the risk of ureteral injury. The procedure continues in the vesicouterine pouch; the dissection plane is angled between the urinary bladder and uterus, preventing thus bladder injury during peritoneal dissection. If this avascular plane, which guarantees dissection without bladder damage, is certainly not identified (sometimes because of adhesions following previous cesarean sections), it is recommended to cut the peritoneum with regular scissors since the possible occurrence of postoperative detachment of tissue which has undergone thermal injury and consequent uroperitoneum being demonstrated [14-16].
At this point of the operation, the dissection of the cervix continues tangent to it and advances at the same time in the ventral plane (between the urinary bladder and uterus). The dissection stops when it is estimated that the portion where the vagina surrounds the cervix has been reached. Now that the right side is completely dissected, the operation continues on the left side.

The surgeon's assistant is asked to steer the uterine manipulator on the other side (in our example to the right of the patient) so that the left flank opens. Dissection via left flank approach is performed in the same manner (Image 5). At this point of the operation, the entire uterus and cervix are completely dissected, the entire structure being now attached only to the vaginal walls. With a monopolar hook or preferably with a monopolar spatula, the anterior vaginal wall is sectioned at maximum $1 \mathrm{~cm}$ from where the vagina

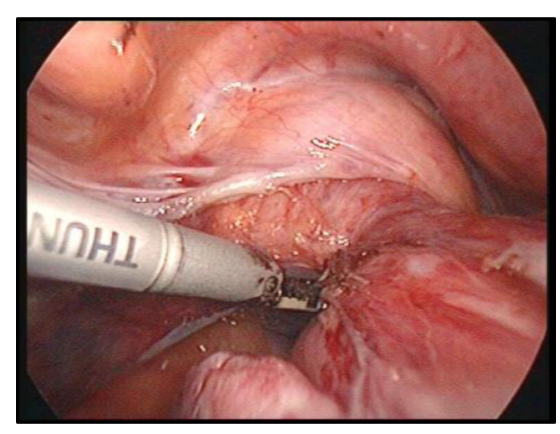

Image 5: Coagulation of the vessels on the left side. Personal collection

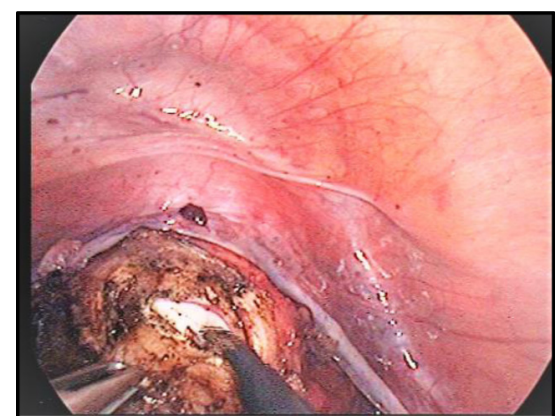

Image 6: Sectioning of the vaginal wall. Personal collection

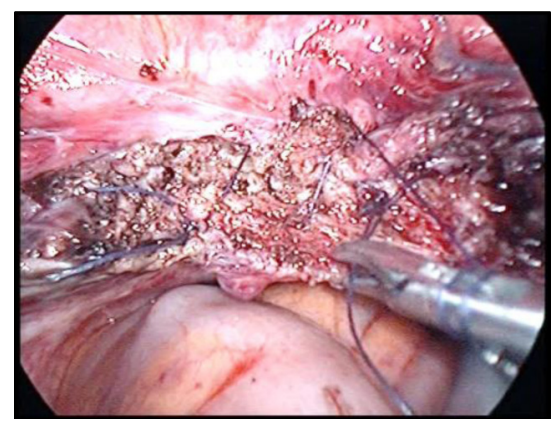

Image 7: Suturing of the vaginal wall. Personal collection 
surrounds the cervix (Image 6). The incision continues until the cap of the manipulator is viewed, making it easier to expose the vaginal insertion, minimizing the risk of injuries to other organs, such as the site where the ureter enters the bladder.

The uterus can be extracted through the vagina or through a trans-parietal incision when larger myomas are being operated. Vaginal cuff hemostasis is then ensured, with the recommendation that coagulation should not occur extensively but only punctually, avoiding thus subsequent vaginal necrosis which usually is a source of important postoperative complications (abscesses, vaginal discharge, vaginal pain). Thereafter, the vagina is sutured intracorporeally using monofilament PDS (polydioxanone) (Image 7). The vaginal cuff may be suspended to the residual uterosacral ligaments.

Uterine morcellation can be tempted in large uteri but it is indicated to use endo-bags (because of oncological reasons) in order to prevent the accidental dissemination of malignant cells that may be present in an undiagnosed sarcoma. A morcellator is a tool that has a cylindrical cutting blade, designed to cut the uterine piece into small fragments inside the abdomen of the patient, which can then be extracted through the trocar of the instrument. It consists of a cylinder which inserts through the trocar and ends with a cutting blade in which the fibroma is inserted, shredded and then extracted by suction outside the abdominal cavity. The development of this instrument has arisen at the beginning of the $90^{\prime}$ as a result of the need for larger volume fibromas to be extracted through small incisions available in minimally invasive surgery [1719]. Although, the FDA (Food and Drug Administration) has issued in 2014 a communication discouraging the use of power morcellation because of a reported case of a woman with fibroma who underwent intraabdominal morcellation, in which the histopathological exam revealed uterine sarcoma and the restaging surgery showed peritoneal metastasis [20]. Taking this into consideration, the European Society of Gynecological Endoscopy (ESGE) has organized a steering committee on fibroid morcellation in order to investigate and evaluate the risk of morcellation [21]. The risk of undiagnosed uterine sarcoma was reported in different studies, but most of them were retrospective or investigated only a few cases, between $0.49 \%$ and $0.056 \%$ [22-25]. The group concluded that there is not enough evidence to estimate the risk in individual patients, the complications of morcellation are rare and prospective data collection may clarify the issue on sarcoma risk in presumed fibroids and that technology of extracting tissue laparoscopically from the abdominal cavity should be perfected.

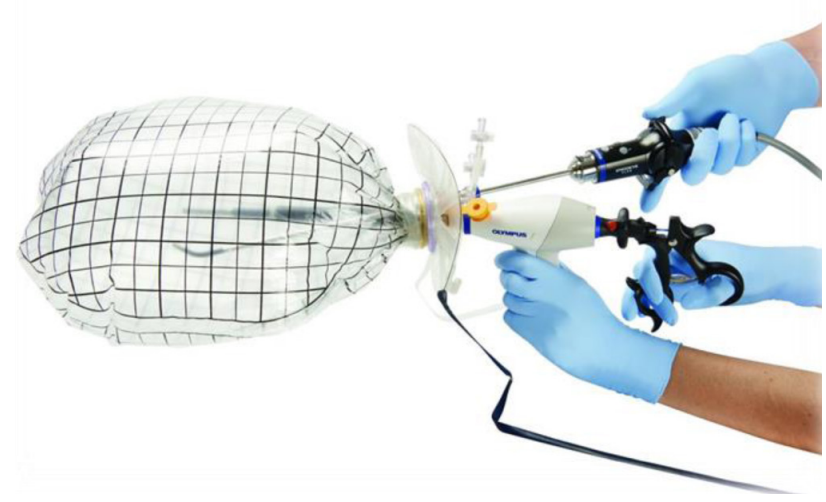

Figure 14: Endo-bag morcellator (Olympus)

Safe endo-bag morcellation (in-bag morcellation) is investigated as it may possibly prevent morcellation complications. Preclinical and clinical results are promising but validating prospective studies are needed in order for in-bag morcellation to be the subject of a consensus recommendation [26-31].

\section{Conclusion}

Analyzing the trend of laparoscopic hysterectomies in Europe, it can be noted that 22 of the 23 Member states of the European Union for which data are available from 2011-2016 reported an increased number of performed hysterectomies; the exception was Slovenia, which reported a decrease in frequency. Four countries reported that the number of laparoscopic hysterectomies doubled; three and four times more laparoscopic hysterectomies were performed in 2016 compared to 2011 in Romania and Hungary, respectively. Despite the increased number of laparoscopic hysterectomies performed between 2011 and 2016, Romania still ranks last in Europe (3\%) [9]. The laparoscopic approach offers the advantages of minimal invasiveness: less pain, faster recovery, and early social reintegration; therefore, this trend of improvement should become more accepted.

\section{Conflict of Interest}

The authors confirm that there are no conflicts of interest.

\section{References}

1. Farquhar $\mathrm{C}$, Steiner $\mathrm{CA}$. Hysterectomy rates in the United States. Obstet. Gynecol. 99,229-234 (2002).

2. Materia E, Rossi L, Spadea $T$ et al. Hysterectomy and socioeconomic position in Italy. J. Epidemiol. Community Health 56,461-465 (2002). 
3. Gimbel H, Settnes A, Tabor A. Hysterectomy on benign indication in Denmark 1988-1998. A register based trend analysis. Acta Obstet Gynecol Scand 2001;80:267-72.

4. Lundholm $C$, Forsgren $C$, Johansson $A L$ et al. Hysterectomy on benign indications in Sweden 1987-2003: a nationwide trend analysis. Acta Obstet Gynecol Scand 2009;88:52-8.

5. Lepine LA, Hilis SD, Marchbanks PA et al. Hysterectomy surveillance -United States, 1980-1993. MMWR CDC Surveill. Summ. 46,1-15 (1997).

6. Wilcox LS, Kononin LM, Pokras R, Strauss LT, Xia Z, Peterson HB. Hysterectomy in the United States, 1988-1990. Obstet. Gynecol. 83,549-555 (1994).

7. Keshavarz $H$. Hysterectomy surveillance - United States, 1994-1999. MMWR Surveill. Summ. 51,1-8 (2002).

8. Jacobson GF, Shaber RE, Armstrong MA, Hung YY. Hysterectomy rates for benign indications. Obstet. Gynecol. 107(6),1278-1283 (2006).

9. https://ec.europa.eu/eurostat/statisticsexplained/index.php/title. Surgical_operations_and_proce-dures

10. Englund M, Robson S Why has the acceptance of laparoscopic hysterectomy been slow? Results of an anonymous survey of Australian gynecologists. J Minim Invasive Gynecol. 2007 NovDec; 14(6):724-8.

11. Treszezamsky AD, Fenske S, Moshier EL, Ascher-Walsh CJ. Neurologic injury and patient displacement in gynecologic laparoscopic surgery using a beanbag and shoulder supports. Int $\mathrm{J}$ Gynaecol Obstet. 2018 Jan; 140(1):26-30.

12. Jon I Einarsson, MPH and Yoko Suzuki, Total Laparoscopic Hysterectomy: 10 Steps Toward a Successful Procedure Rev Obstet Gynecol. 2009 Winter; 2(1): 57-64.

13. Hurd W, Falcone, Rivlin M. Gynecologic Laparoscopy Treatment and Management. https://emedicine.medscape.com/article/265201treatment

14. V.P. Lamaro, J.D. Broome, T.G. Vancaillie Unrecognized bladder perforation during operative laparoscopy. J. Am. Assoc. Gynecol. Laparosc. 7(3) (2000), pp. 417-419

15. A. Ostrzenski, K.M. Ostrzenska Bladder injury during laparoscopic surgery Obstet. Gynecol. Surv., 53 (3) (1998), pp. 175-180

16. Dawkins J.C, Lewis G.K, Christensen B, Wortman M. Urinary ascites in late onset of bladder injury post laparoscopic hysterectomy. Case Rep Womens Health. 2017 Sep 22;16:8-10

17. Steiner RA, Wight E, Tadir $Y$, Haller U. Electrical cutting device for laparoscopic removal of tissue from the abdominal cavity. Obstet Gynecol. 1993 Mar; 81(3):471-4.

18. Morcellator, Jude S. Sauer, Roger J. Greenwald, Mark A. Bovard, John F. Hammond - US Patent 5562694. Issue date: Oct 8, 1996.

19. Disposable laparoscopic morcellator, GM Savage, JJ Christian, DC Dillow - US Patent 6,039,748, 2000
20. FDA, FDA discourages use of laparoscopic power morcellation for removal of uterus or uterine fibroids. Food Drug Adm. 2014;17:4.

21. Hans Brölmann, Vasilios Tanos, Grigoris Grimbizis, Thomas Ind, Kevin Philips, Thierry van den Bosch, Samir Sawalhe, Lukas van den Haak, Frank-Willem Jansen, Johanna Pijnenborg, FlorinAndrei Taran, Sara Brucker, Arnaud Wattiez, Rudi Campo, Peter O'Donovan, Rudy Leon de Wilde, and On behalf of the European Society of Gynaecological Endoscopy (ESGE) steering committee on fibroid morcellation. Options on fibroid morcellation: a literature review. Gynecol Surg. 2015; 12(1): 3-15.

22. Rowland M, Lesnoch J, Edwards R, Richard S, Zom K, Sukumvanich P. Occult uterine cancer in patients undergoing laparoscopic hysterectomy with morcellation. Gynecol Oncol. 2012;127(1): S29.

23. Bojahr B, De Wilde RL, Tchartchian G. Malignancy rate of 10,731 uteri morcellated during laparoscopic supracervical hysterectomy (LASH). Arch Gynecol Obstet. 2015 Sep; 292(3):665-72.

24. Harlow BL, Weiss NS, Lofton S. The epidemiology of sarcomas of the uterus. J Natl Cancer Inst. 1986 Mar; 76(3):399-402.

25. Multinu F, Casarin J, Tortorella L, Huang Y, Weaver A, Angioni S, Melis GB, Mariani A, Stewart EA, Laughlin-Tommaso SK. Incidence of sarcoma in patients undergoing hysterectomy for benign indications: a population-based study. Am J Obstet Gynecol. 2019 Feb;220(2):179.e1-179.

26. K.A. Shibley, Feasibility of Intra-Abdominal Tissue Isolation and Extraction, within an Artificially Created Pneumoperitoneum, at Laparoscopy for Gynecologic Procedures. J Minim Invasive Gynecol. 2012;19[6]:[Suppl] S75.

27. Anapolski M, Panayotopoulos D, Alkatout I, Soltesz S, Mettler L, Schiermeier S, Hatzmann W, Noé G. Power morcellation inside a secure endobag: a pilot study. Minim Invasive Ther Allied Technol. 2016 Aug;25(4):203-9.

28. Anapolski M, Panayotopoulos D, Alkatout I, Soltesz S, Schiermeier $\mathrm{S}$, Noé G. Preclinical safety testing for morcellation and extraction for an endobag with sealable ports: in vitro pilot study. Surg Endosc. 2017 Jan;31(1):494-500.

29. Devassy R, Cezar C, Krentel H, Verhoeven HC, Devassy R, de Wilde MS, Torres-de la Roche LA, de Wilde RL. Feasibility of myomatous tissue extraction in laparoscopic surgery by contained in - bag morcellation: A retrospective single arm study. Int J Surg. 2019 Feb;62:22-27.

30. Favero G. Tips and tricks for successful manual morcellation: a response to "vaginal morcellation: a new strategy for large gynecological malignant tumors extraction. A pilot study". Gynecol Oncol. 2013 Jan; 128(1):151.

31. ACOG Committee Opinion No. 770: Uterine Morcellation for Presumed Leiomyomas. Obstet Gynecol. 2019 Mar;133(3): e238-e248. 